\title{
Modeling of the residual kinematic errors of coordinate measuring machines using LaserTracer system
}

\author{
Adam Gąska • Marcin Krawczyk • Robert Kupiec • \\ Ksenia Ostrowska $\cdot$ Piotr Gąska $\cdot$ Jerzy Sladek
}

Received: 29 August 2013 /Accepted: 1 April 2014 / Published online: 25 April 2014

(C) The Author(s) 2014. This article is published with open access at Springerlink.com

\begin{abstract}
The following article examines the methodology of identifying and modeling kinematic errors of coordinate measuring machines with the use of a modern and precise interferometric system - the LaserTracer. Since the kinematic errors of coordinate measuring machines (CMMs) are by far the most prevalent factor in measurement uncertainty, a specially developed model could be used for efficient uncertainty evaluation as an additional module of the virtual measuring machine responsible for simulation of the CMM kinematic errors. The model could also be of use for determining optimal measurement strategy, and would potentially improve the uncertainty of measurements performed.
\end{abstract}

Keywords CMM $\cdot$ LaserTracer · Accuracy $\cdot$ Monte Carlo method

\section{Introduction}

One of the most important problems a metrologist must face involves the correct assessment of measurement uncertainty, which can be regarded as a quantitative representation of measurement accuracy. It is commonly known that the result of any measurement given without the corresponding uncertainty is very unreliable and offers little practical value. However, in the case of coordinate measuring techniques (CMT), determining measurement uncertainty may be particularly difficult, and the results may not be very straightforward [1-4]. It is a common practice among metrologists (as well as the manufacturers of measuring systems) to circumvent the

A. Gąska $(\bowtie) \cdot$ M. Krawczyk $\cdot$ R. Kupiec $\cdot$ K. Ostrowska P. Gaska · J. Sładek

Laboratory of Coordinate Metrology, Cracow University of

Technology, al. Jana Pawla II 37, 31-864 Cracow, Poland

e-mail: agaska@mech.pk.edu.pl problem by providing the accuracy of the measuring devices rather than individual measurements. This approach describes accuracy in relation to the maximum permissible error (MPE), and the measurement itself is regarded as a measurement of distance, which seems to be a significant oversimplification for the majority of tasks. In most cases, measurement accuracy determined in this way tends to vary significantly from the accuracy of a real measurement task, and may lead to incorrect conclusions as far as compliance with the specifications of a given product is concerned [2, 5-11]. Therefore, it is crucial to employ more reliable and metrologically validated methods for coordinate measurement uncertainty estimation.

One of the primary factors affecting measurement accuracy involves errors of the measuring machine's kinematic system [12]. The initial models of the kinematic errors of coordinate measuring machines (CMMs) were created and implemented during the 1970s [13], but the very first attempts aimed at eliminating the geometric errors of machine tools date back to as early as the second half of the nineteenth century $[14,15]$. However, the first attempts of eliminating the machine tools geometric errors were made at the second half of nineteenth century $[14,15]$. Nowadays, in the era of cost minimization, the vast majority of measuring and machining devices is equipped with their own geometric error software correction systems, because it is generally more profitable to massproduce parts (which form the kinematic system of machine) which are more distant from the ideal geometry, and to compensate the geometric errors influenced by these faults rather than to produce expensive parts with very narrow shape and dimension tolerances.

There are only a few available models of CMM errors (i.e., full rigid body, reduced rigid body) which are used to determine the geometrical errors described by a varying number of geometrical components. More often, models are supplemented with elastic errors of the machine. The most commonly encountered model consists of 21 geometric error components, 
which includes translation, rotation, and squareness errors [16]. Based on that, it can be stated that the current CMM models regard kinematic errors only as the source of systematic errors, and identify their vales in order to correct them mathematically. This paper presents a possible expansion of the models currently in use. The described approach involves the assumption that the systematic parts of CMM kinematic errors are properly compensated using one of the mentioned models, and shows the method for assessing the impact of uncompensated kinematic errors.

In most contemporary CMMs, the analysis of the distribution of kinematic errors is performed by the manufacturer during the development of the computer-aided accuracy (CAA) correction matrix. As a result, the end user receives a product for which the impact of geometric errors on measurement accuracy is already partly reduced. However, not all kinematic errors can be corrected in this way. Residual errors which remain uncompensated after the correction process may also influence the accuracy of measurements. Therefore, it is important to identify the field of these errors. This article describes the methodology for identifying and modeling residual errors associated with the kinematic system of CMMs.

A specially designed model, the so-called virtual measuring machine (VCMM), can be used as part of an online system for assessing the accuracy of coordinate measurements. This method is based on identifying the factors affecting measurement accuracy, designation of their variability and, finally, simulation of their value. The components affecting measurement uncertainty which are usually taken into account are kinematic system errors of the CMM, probe head errors, influences of measured object, and other types of errors influenced by the ambient conditions. These components may be successfully simulated by specially created modules. The values obtained from each module may then be added up to determine the total measurement uncertainty. Many interesting solutions for VCMM design are discussed in [17-33]. Additionally, the described model could also be of aid in determining the optimal measurement strategy for each project. Examples of such application will also be presented in this article.

\section{The concept of identifying and modeling of CMM kinematic errors}

The first concepts of modeling of CMM kinematic errors relied upon the designation of individual components of the measuring machine geometric errors (21 components) at different points in the measuring volume. The hole-plate standards were most commonly used for this purpose. The standards were repeatedly measured at different positions so as to cover the maximum possible part of the machine's measuring volume. This concept is described at length in $[18,32,33]$. A more recent approach involves the use of the matrix method which describes the vector character of errors [29, 34]. The matrix method employs a grid of reference points and describes points located in the nodes of the grid by assigning a vector expressing the error of measuring point reproducibility. Quite expectedly, the kinematic errors constitute a significant subset of all errors in this method. Up to this point, the measuring point reproducibility errors were modeled through a grid of reference points describing the machine's measuring volume, in which the values of components affecting this error were determined. These components were then divided into components of a systematic and random character. The systematic components were caused by the kinematic errors of the machine, whereas random errors signified other unidentified errors influencing the reproduction of measuring point. In this article, the authors present a new method for describing the field of CMM kinematic errors.

Bearing in mind that in most CMM kinematic errors are compensated by the correction matrix and that most machines are equipped with their own temperature compensation systems, it comes as a natural conclusion that the inaccurate reproduction of the measuring point must, in fact, be attributed to the residual and random errors. Figure 1 shows a schematic grid of reference points within the distribution of probability (the $t$ distribution seems to work best in this context), as reproduced by the machine. However, it is still necessary to construct a system which would be able to determine the distribution of errors in reference points experimentally, and within required accuracy. The lack of such systems in place seems to constitute a major obstacle for a successful implementation of the concept described previously. One specific device which satisfies those requirements is the LaserTracer (LT) developed by Etalon AG.

LaserTracer is an interferometric device that allows for tracking of reflector movements. The reflector is usually mounted on the probe head of a measuring device or on the tool holder of a machine tool. The uncertainty of length measurement of this device is presented in Eq. 1:

$U=0.2 \pm 0.3 * L / 1,000 \mu \mathrm{m}$

where $L$ is the measured value given in mm.

Such high level of accuracy can be achieved; thanks to the implementation of novel solutions used in the construction process of the device whose form errors do not exceed $30 \mathrm{~nm}$. The reference sphere is located in the center of rotation of the LT rotary system. A schematic of the LaserTracer is presented in Fig. 2.

In LaserTracer, the interferometer moves on a gimbal mount around a fixed precision sphere serving as a reference mirror which reflects the laser beam, and radial and lateral deviations of the mechanical rotation axes do not have a 
Fig. 1 a Grid of reference points. b Residual errors (in nodes of reference grid) and method of their identification
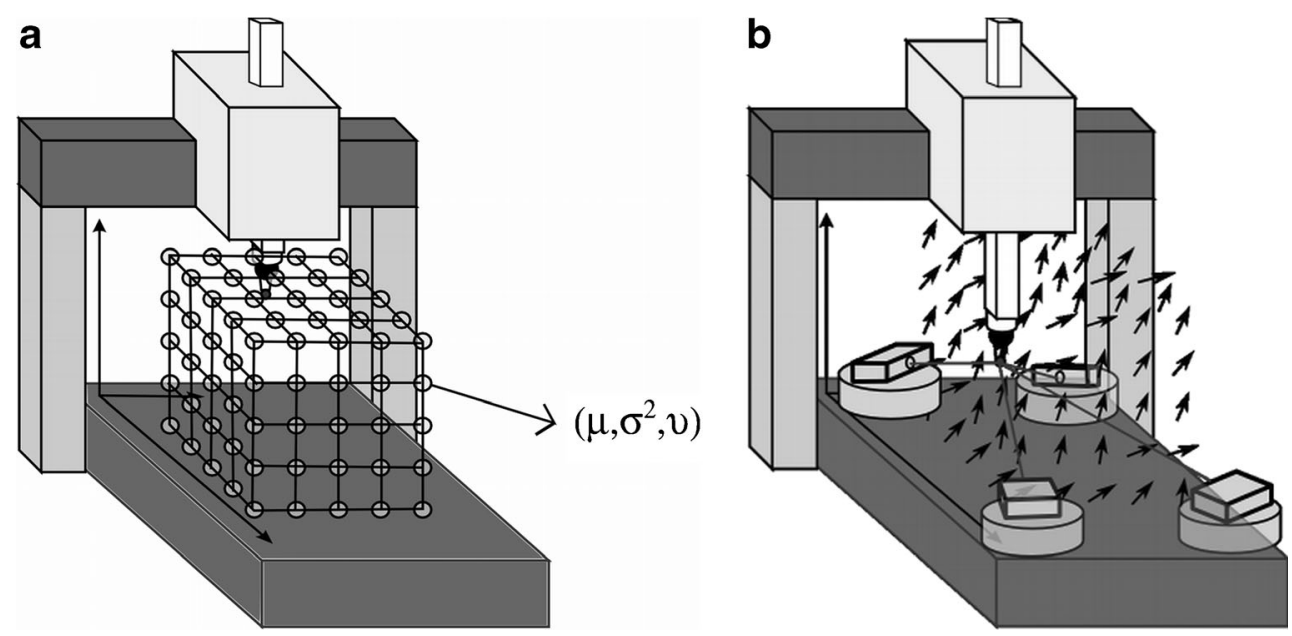

significant effect on measurement accuracy [35]. It is also important to observe that the LaserTracer does only measure the distance from device to reflector, which indicates that it is still necessary to use a multilateration technique in order to determine the coordinates of a measuring point.

The LaserTracer allows to determine the probability of reproduction of the measuring point, as well as probability distribution, by showing the distribution of residual errors for the entire measuring space of the CMM.

Once the grid of reference points is described (Table 1), the problem of grid compaction remains to be tackled. It is known that during measurements, the majority of points described is found between nodes of the grid. Thus, the strategy for grid condensation should be selected in a way which would allow to simulate (through the use of the Monte Carlo method) achieving all possible points within the measuring space. There are several ways to do this, e.g., the method proposed in [29], which suggests the application of a neural network for this purpose. The authors of this article have developed an alternative method, which relies on the use of $b$-spline curves in conjunction with the "nearest neighbor" or bilinear interpolation methods, implemented according to Monte Carlo method simulations. A detailed description of this method will be discussed later on in this article.

\section{Identification of residual errors}

Adopting the assumptions described in the previous chapter, the residual and random errors were estimated (Fig. 4, Table 2). Together, these errors form the starting point for developing a model of the CMM kinematic errors. As mentioned previously, this model is based on the concept of the measuring point reproducibility error. To successfully model these errors, it is necessary to create a grid of reference points which would be linked closely to the grid on which the machine correction matrix is described. It is also necessary to assume a degree of overlapping between nodes of the reference grid and the correction matrix as dictated by the concept of the model. The model remains in an active connection with the correction matrix and uses residual and random errors as its computational basis. This allows
Fig. 2 LaserTracer: 1 reference sphere, 2 laser beam, 3 LT column (www.etalon-ag.com)

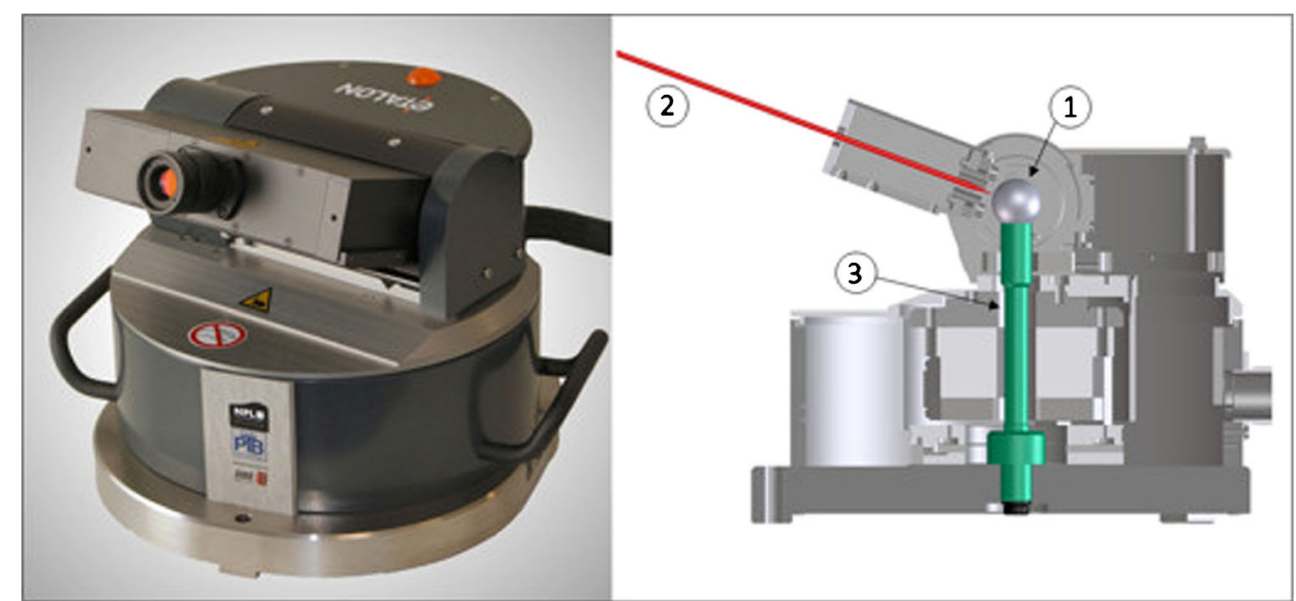


Table 1 Nominal coordinates of reference points (when the modeled area has dimensions of 700:600:500 mm and with the 150-, 50-, and 50-mm point taken as the starting point)

\begin{tabular}{|c|c|c|c|c|c|c|c|c|c|c|c|c|c|c|c|}
\hline No. & $X$ & $Y$ & $Z$ & No. & $X$ & $Y$ & $Z$ & No. & $X$ & $Y$ & $Z$ & No. & $X$ & $Y$ & $Z$ \\
\hline 1 & 150 & 50 & 50 & 14 & 310 & 460 & 200 & 27 & 475 & 325 & 350 & 40 & 640 & 190 & 500 \\
\hline 2 & 150 & 50 & 200 & 15 & 310 & 460 & 350 & 28 & 475 & 325 & 500 & 41 & 800 & 50 & 50 \\
\hline 3 & 150 & 50 & 350 & 16 & 310 & 460 & 500 & 29 & 475 & 600 & 50 & 42 & 800 & 50 & 200 \\
\hline 4 & 150 & 50 & 500 & 17 & 310 & 190 & 50 & 30 & 475 & 600 & 200 & 43 & 800 & 50 & 350 \\
\hline 5 & 150 & 325 & 50 & 18 & 310 & 190 & 200 & 31 & 475 & 600 & 350 & 44 & 800 & 50 & 500 \\
\hline 6 & 150 & 325 & 200 & 19 & 310 & 190 & 350 & 32 & 475 & 600 & 500 & 45 & 800 & 325 & 50 \\
\hline 7 & 150 & 325 & 350 & 20 & 310 & 190 & 500 & 33 & 640 & 460 & 50 & 46 & 800 & 325 & 200 \\
\hline 8 & 150 & 325 & 500 & 21 & 475 & 50 & 50 & 34 & 640 & 460 & 200 & 47 & 800 & 325 & 350 \\
\hline 9 & 150 & 600 & 50 & 22 & 475 & 50 & 200 & 35 & 640 & 460 & 350 & 48 & 800 & 325 & 500 \\
\hline 10 & 150 & 600 & 200 & 23 & 475 & 50 & 350 & 36 & 640 & 460 & 500 & 49 & 800 & 600 & 50 \\
\hline 11 & 150 & 600 & 350 & 24 & 475 & 50 & 500 & 37 & 640 & 190 & 50 & 50 & 800 & 600 & 200 \\
\hline 12 & 150 & 600 & 500 & 25 & 475 & 325 & 50 & 38 & 640 & 190 & 200 & 51 & 800 & 600 & 350 \\
\hline 13 & 310 & 460 & 50 & 26 & 475 & 325 & 200 & 39 & 640 & 190 & 350 & 52 & 800 & 600 & 500 \\
\hline
\end{tabular}

modification of the model depending on the matrices obtained in the future and also performing the interpolation based on the same nodes.

The number of reference points should be selected in such a way so as to cover the entire measuring space of the machine evenly, bearing in mind that a greater number of reference points would also mean more time and effort spent on developing the model without necessarily yielding more accurate results. The authors created a grid consisting of 52 reference points in the measuring space of the machine. This number of points is sufficient to faithfully reproduce the field of residual and random errors, as shown in [34].

The values of random errors for points located in the nodes of the reference grid were determined experimentally through repeated approaching at each point from different directions. The measuring machine used for the task was Leitz PMM 12106. Instead of using the default Leitz stylus, a "cateye" retroreflector was mounted (Fig. 3).

The position of the retroreflector was tracked in dynamic mode by a LaserTracer installed in the measuring volume of the machine. For each measuring point, the sequence consisting of 14 approaches was performed (and distance from LT was measured): six in directions consistent with the three axes of the machine (with negative and positive orientation) and eight in direction inclined at a $45^{\circ}$ angle to each axis (with negative and positive orientation). After each sequence of approaches at the point, the machine reached the next one and the cycle was repeated, in order to carry out the sequence for all points in the nodes of the reference grid. The nominal coordinates of all reference points are displayed in Table 1.

The described measuring path was repeated five times with the position of the LT being changed every time. With the use of the multilateration method, the final coordinates of the nodes (14 sets of coordinates for one point) were determined. The coordinates of these points were determined according to Eq. 2 [36]:

$W_{i j}=l_{i j}+l_{0 j}-\sqrt{\left(x_{i}-X_{0 j}\right)^{2}+\left(y_{i}-Y_{0 j}\right)^{2}+\left(z_{i}-Z_{0 j}\right)^{2}}$

where

a $i$ - the number of the $i$ th point; $j$-number of the $j$ th position of LaserTracer; $w_{i j}$-minimized residue; $l_{i j}$-distance to the $i$ th point measured by LaserTracer located in the $j$ th position; $l_{0 j}$ - "dead path" length for LaserTracer at $j$ th

Table 2 The results of measurements presented for selected points: $X, Y, Z$-nominal coordinates of reference points, $\mathrm{mm} ; d(X), d(Y), d(Z)-$ standard deviations of reproduction of certain coordinates of points calculated from results for all approaches at the point, $\mathrm{mm}$

\begin{tabular}{llllllllllllll}
\hline Lp. & $X$ & $Y$ & $Z$ & $d(X)$ & $d(Y)$ & $d(Z)$ & Lp. & $X$ & $Y$ & $Z$ & $d(X)$ & $d(Y)$ & $d(Z)$ \\
\hline 1 & 310 & 190 & 50 & 0.00063 & 0.00066 & 0.00065 & 5 & 475 & 50 & 50 & 0.00212 & 0.00169 & 0.00097 \\
2 & 310 & 190 & 200 & 0.00020 & 0.00023 & 0.00089 & 6 & 475 & 50 & 200 & 0.00101 & 0.00073 & 0.00058 \\
3 & 310 & 190 & 350 & 0.00023 & 0.00053 & 0.00127 & 7 & 475 & 50 & 350 & 0.00118 & 0.00070 & 0.00061 \\
4 & 310 & 190 & 500 & 0.00025 & 0.00043 & 0.00046 & 8 & 475 & 50 & 500 & 0.00129 & 0.00092 & 0.00021 \\
\hline
\end{tabular}




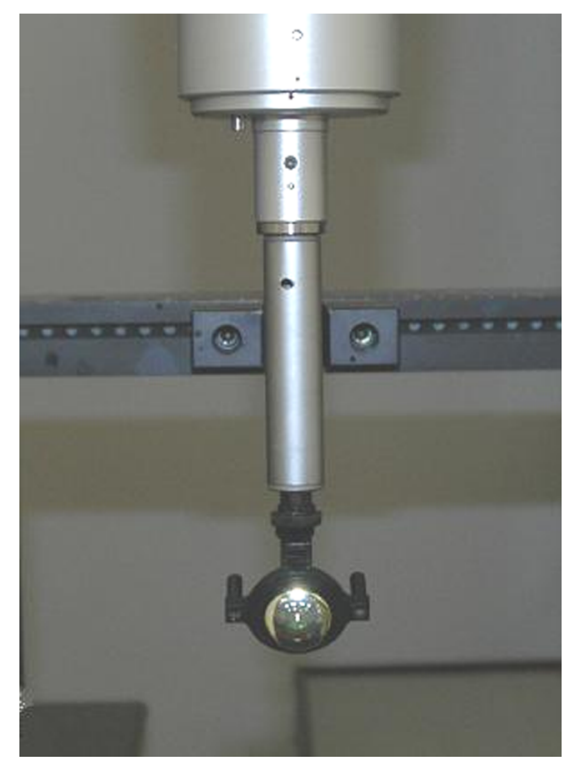

Fig. 3 "Cateye" retroreflector mounted on measuring machine

position; $x_{i}, y_{i}, z_{i}$-coordinates of $i$ th point; and $X_{0 j}, Y_{0 j}, Z_{0 j}-$ coordinates of LaserTracer in $j$ th position.

The final value of coordinates for each reference point is obtained by minimizing the sum of squared residues $w_{i j}$. The results of the analysis performed are shown in Fig. 4 and Table 2.

The maximum values of the deviations (difference between nominal and actual coordinates) in directions of each axis were as follows: $d_{\max }(X)=0.0026 \mathrm{~mm}, d_{\max }(Y)=$ $0.0018 \mathrm{~mm}, d_{\max }(Z)=0.0015 \mathrm{~mm}$; mean value of deviations, $\bar{d}(Z)=0.0009 \mathrm{~mm}, \bar{d}(Y)=0.0008 \mathrm{~mm}, \bar{d}(Z)=$ $0.0007 \mathrm{~mm}$. The data obtained in accordance with the described methodology provide the basis for functioning of the model of CMM kinematic errors [34, 36].

\section{Modeling of kinematic system residual errors}

The primary purpose of the described model is to determine the variability of reproduction of each point with its own set of coordinates, which can be expressed as a standard deviation of point coordinates found by performing repeated approaching on the points located in the nodes of the reference grid. Then, the reproduction of each point is simulated with the use of a Monte Carlo simulation. The Monte Carlo method was chosen for this purpose not because of complexity of numerical problem but because of its suitability for dealing with repeated random samplings of points with varying probability distribution.

The reproduction variability for points located in the nodes of the reference grid can be determined experimentally, as described in sections 2 and 3. In the case described, the simulation is performed on data corresponding to reference points taken directly from the input matrix of the model, which contains the following values (sorted in rows): $X, Y, Z$ nominal point coordinates, $d(X), d(Y)$, and $d(Z)$ standard reproduction deviations for each coordinate. This simulation covers only a small subset of possibilities considering the total number of points included in measurements. It is also known that most of the points will be located between the nodes of the reference grid. This necessitates further use of interpolation methods, in addition to the Monte Carlo simulation.

The first step in the interpolation method is to form threedimensional $b$-spline functions describing the variability of coordinate values for reference points situated in the direction of the $z$ axis (as shown in Table 1; reference points are arranged in vertical columns consisting of four points). Therefore, $13 b$-spline curves have to be created; each spread over four reference points (an example of such curve, spread over points nos. 17-20 from Table 1, is shown in Fig. 5). The

Fig. 4 Ellipsoids of point reproduction error

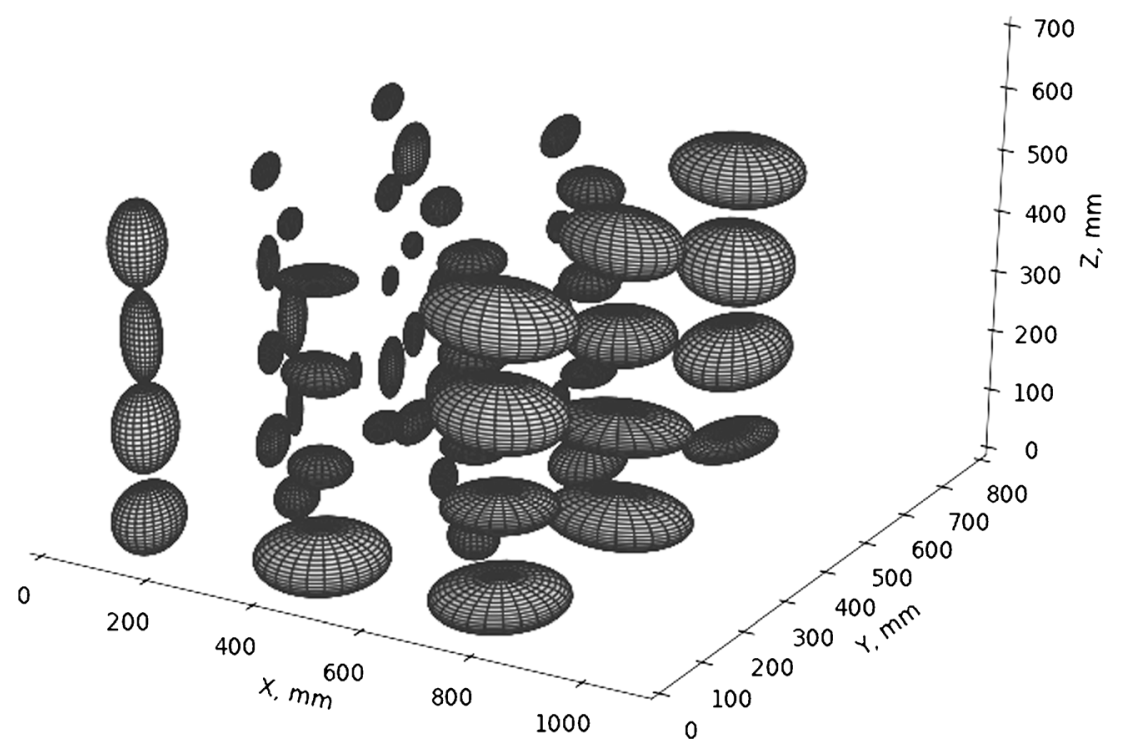


Fig. 5 One of the 13 threedimensional $b$-spline functions spread over four reference points (top view-upper left corner, front view-upper right corner, and side view-lower left corner). This $b$-spline function was achieved for reference point nos. 17, 18, 19, and 20 in Table 1
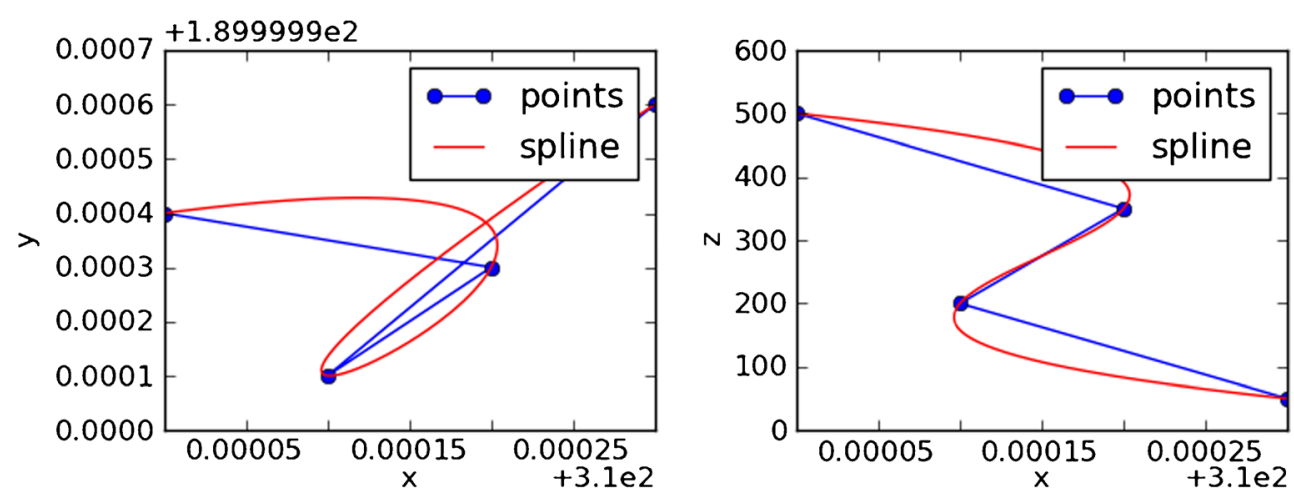

values of reference points coordinates are simulated by Monte Carlo method using the scaled and shifted $t$ distribution. To each reference point in the measuring volume of the machine, a particular probability distribution function is assigned. For all points, the $t$ distribution with parameters $(\bar{x}, \sigma, v)$ is assigned, where $\bar{x}$ is the mean value of the distribution, $\sigma$ standard deviation, and $v$ the number of degrees of freedom. As the mean value, the nominal values of coordinates of each point are assumed, as the standard deviation; the previously mentioned values of $d(X), d(Y)$, and $d(Z)$ are taken; the number of degrees of freedom is set for each of the points as 13 , because it was assumed that the number of degrees of freedom is equal to the number of measurements for each point minus one 14-1=13 (the number of measurements and measurement procedure are explained in Sect. 3). For 10 out of 52 reference points, a chi-squared test was performed for the distribution of $x, y$, and $z$ coordinate reproduction. All of these tests indicated no reason for rejecting the null hypothesis regarding the $t$ character of distribution.

The next step is to intersect all of the $b$-spline curves with a plane parallel to the XY plane, whose distance from this plane is equal to $z_{1}$, where $z_{1}$ is the $z$ coordinate of the simulated point. The parallel plane intersects each of the $b$-spline curves, thus creating 13 points for which the values of $(x, y, z)$ coordinates are known. These points of intersection form the basis for interpolation according to the chosen principle. The applicability of both possible interpolation methods (bilinear interpolation and "nearest neighbor") is further discussed in Sect. 5. Both interpolation methods assume that the $f(x, y)$ function is defined, and its values are known for the $n$-selected nodes. With those assumptions in place, it is then possible to interpolate values of the $f$ function in any place belonging to the function domain and located between the selected nodes. In the "nearest neighbor" method, the interpolating value of the specified point is the value copied from a node that is closest to that point. For bilinear interpolation, the interpolated value of a specified point is calculated by taking the values of the function in nodes surrounding the point considered, and performing linear interpolation in two directions (in the direction of the $x$ and $y$ axes of the coordinate system).

By applying one of these methods, it is possible to interpolate the values describing the $x$ coordinate (using the $x$ coordinate values defined in relation to the $(y, z)$ coordinates as input values in selected interpolation method) and the $y$ coordinate (by taking the $y$ coordinate values defined in relation to the $(x, z)$ coordinates as input values in selected interpolation method). The value of the $z$ coordinate is obtained as a result of Monte Carlo simulations of the $z$ coordinate value for the node on the reference grid which is the closest to considered point. It is impossible to simulate the $z$ coordinate value in the same way as for the $x$ and $y$ coordinates because the previous step of the 
simulation assumes that the $z$ coordinate of the intersecting plane is taken as a constant.

The obtained $\left(x_{1}, y_{1}, z_{1}\right)$ coordinates of the point are then simulated $n$ times, where $n$ is the number of Monte Carlo simulations. A schematic algorithm of the residual error simulation process for a single measuring point is shown in Fig. 6 .

The proposed model of kinematic error simulation can be used as part of VCMM. Further examples proving its possible application for this task can be found in $[34,36]$. The model could also be of use for determining the optimal position of a measured workpiece in the measuring volume of the machine. An optimal position indicates the position for which the uncertainty of measurement is reduced.

\section{Determining the optimal position of a measured workpiece in the measuring volume}

For the purposes of the experiment presented below, it is assumed that the workpiece is going to be measured using the same probing points and measuring program for all of its positions. If the ambient conditions remain stable, it can be concluded that differences in the uncertainties of measurements performed in different positions in the measuring volume of the machine are caused by kinematic errors of the machine. Using the CAA compensation matrix, it is then possible to determine that the residual errors of the machine are a primary factor in uncertainty differences. Leitz PMM 12106 was used for all experiments presented in this section. The machine is installed in a strictly air-conditioned room (temperature variation in the measuring volume of the machine is $\pm 0.05{ }^{\circ} \mathrm{C}$ ), which provides very good stabilization of ambient conditions.

Figure 7 (found below) illustrates the simulated values for the reproduction error of coordinates (for each coordinate separately) obtained through the simulations described previously in Sect. 4. For the $x$ and $y$ coordinates, the results of simulation were shown for both possible interpolation methods: the "nearest neighbor" method and bilinear

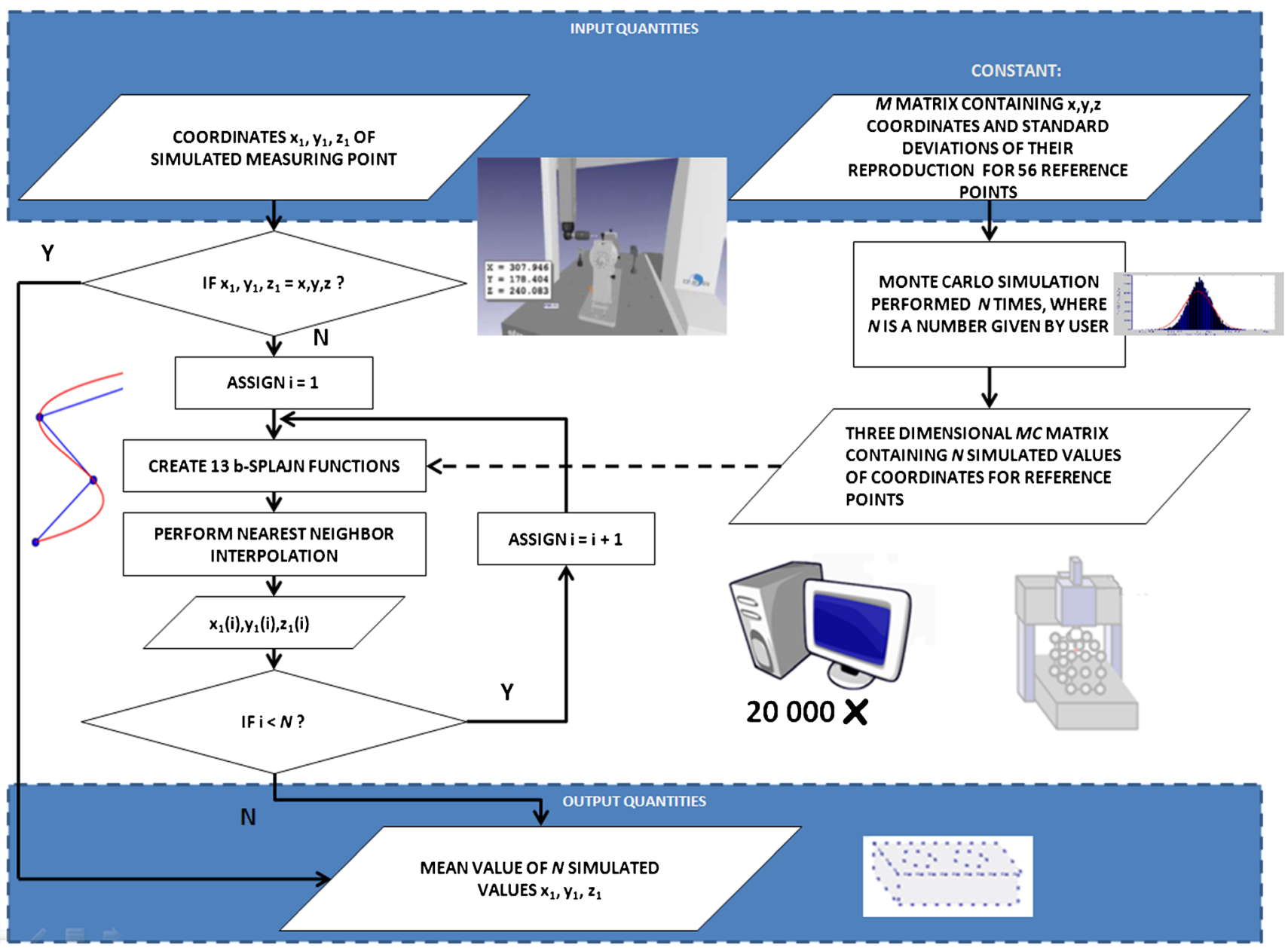

Fig. 6 Schematic algorithm of residual error simulation process when nearest neighbor interpolation is used 

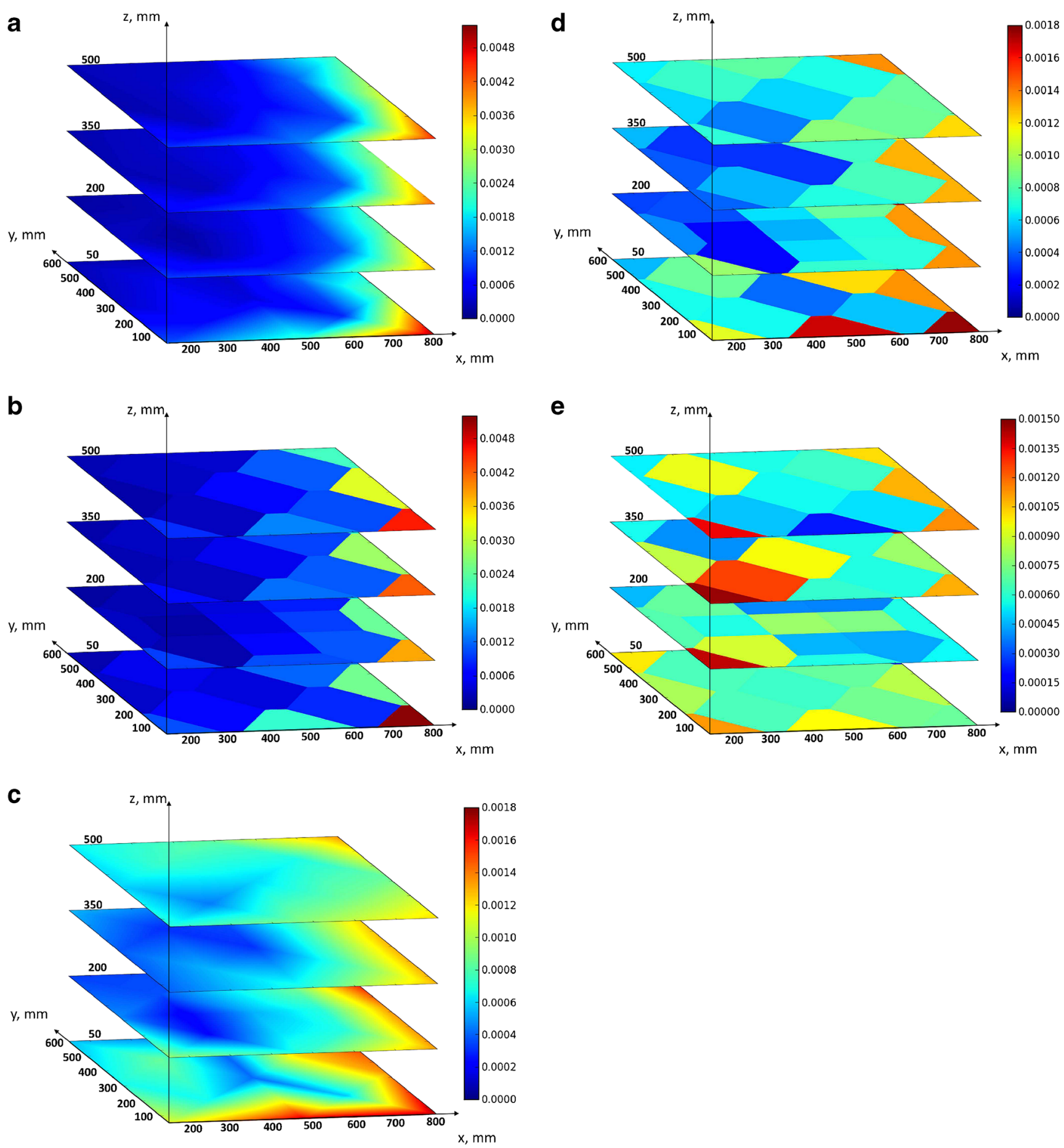

Fig. 7 Results of simulation of coordinate reproduction error values. a For the $x$ coordinate obtained using bilinear interpolation. b For the $x$ coordinate obtained using "nearest neighbor" interpolation. $\mathbf{c}$ For the $y$

interpolation. For the $z$ coordinate, only one method of interpolation is used ("nearest neighbor").

The experiment consisted of repeated measurements of a compressor body (shown in Fig. 8) placed in various positions in the measuring volume of the machine. The same sampling

coordinate obtained using bilinear interpolation. d For the $y$ coordinate obtained using "nearest neighbor" interpolation. e For the $z$ coordinate obtained using "nearest neighbor" interpolation

strategy was used for all positions. In this case, the errors of the probe head (which are mainly dependent on the direction of approach) can be treated as being the same for corresponding measuring points (in different positions of the measured object). Because of that, the probe head errors would have the 


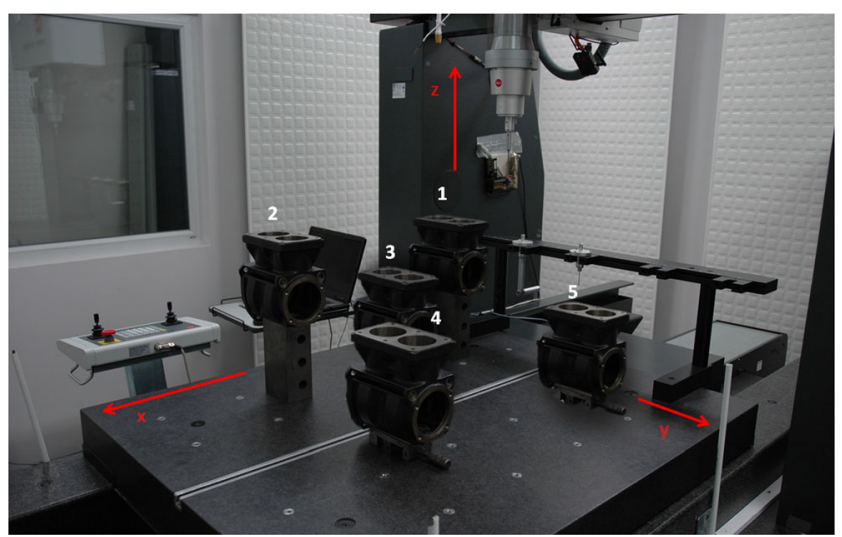

Fig. 8 Different positions of the workpiece during measurements performed on a PMM machine. Red arrows indicate directions of machine coordinate system axes

same influence on all of the measurements regardless of position of the measured body. The form error of one of the cylinders and the distance between the front planes of the body were measured for each respective position. All measurements were conducted according to [37], and for each position, the results of measurement, and the corresponding uncertainty, were calculated. The standards used during measurements were the Koba-step and the ring-shaped internal diameter standard.

Detailed results of measurements are presented in Table 3. By analyzing Fig. 7, it can be observed that the lowest values of coordinate reproduction errors were achieved for positions 3 and 5. Based on these observations, it can be concluded that it would be possible to determine the optimal position of the measured workpiece by utilizing diagrams used in the described model.

The differences between uncertainty values shown in Table 3 are relatively small (differences do not exceed $0.4 \mu \mathrm{m})$. However, for high-end machines like the PMM 12106 , such scale of differences can be regarded as significant, especially if the machine is used for performing calibration measurements in an accredited calibration laboratory. What is more, the differences in uncertainty values correlate with changes in the coordinate reproduction errors shown in Fig. 7.

\section{Conclusion}

The presented model of kinematic errors focuses only on the simulation of random errors, and assumes that the systematic errors are already compensated. This approach, as demonstrated in the experiments developed and conducted by the authors, reduces the time needed for identifying kinematic errors throughout the volume of the machine. Thanks to the modern system LaserTracer (used for the first time for residual errors modeling) and the Trac-Cal software; all data necessary to build the model can be collected within $6 \mathrm{~h}$, which is less than the duration of a standard factory shift.

Furthermore, the model can be successfully used as part of a measurement uncertainty evaluation system. It can also be used for determining the optimal position of a measured workpiece on the machine volume in order to reduce measurement uncertainty. The idea of uncertainty reduction is based around the fact that the random errors cannot be compensated, which indicates that in order to reduce their influence on measurement uncertainty, it is necessary to identify the area in which they are significantly smaller. This function could be extremely important for high-accuracy coordinate measuring machines used in calibration laboratories, where it is crucial to reduce measurement uncertainty even by the tiniest fraction of a micron. As far as directions for further research are concerned, subsequent experiments aiming to reduce the amount of data needed to create a model of the machine's residual errors have to be performed. The authors are fully convinced that it should be possible to further reduce the number of reference points required. However, the remaining question is whether and how a reduced number of reference points would affect the correct function of the model.

The optimal position of a measured workpiece is currently determined only through a graphical analysis of the simulated residual errors. However, it is certainly possible to create an application which would automatically detect the optimal placement of a measured workpiece using the discussed model and additional data concerning the shape of the object. It could also be possible to combine this application with the module responsible for the simulation of probe head errors (like the one presented in [36]), which would allow to limit

Table 3 Results of the measurements performed

\begin{tabular}{|c|c|c|c|c|c|c|c|c|c|c|}
\hline \multirow[t]{2}{*}{ Measured feature } & \multicolumn{2}{|c|}{ Localization 1} & \multicolumn{2}{|c|}{ Localization 2} & \multicolumn{2}{|c|}{ Localization 3} & \multicolumn{2}{|c|}{ Localization 4} & \multicolumn{2}{|c|}{ Localization 5} \\
\hline & Result & $U$ & Result & $U$ & Result & $U$ & Result & $U$ & Result & $U$ \\
\hline Form error & 0.0117 & 0.0006 & 0.0116 & 0.0008 & 0.0114 & 0.0005 & 0.0113 & 0.0009 & 0.0116 & 0.0005 \\
\hline Distance & 188.9299 & 0.0004 & 188.9295 & 0.0005 & 188.9298 & 0.0004 & 188.9292 & 0.0006 & 188.9297 & 0.0003 \\
\hline
\end{tabular}

All values are given in $\mathrm{mm}$ 
measurement uncertainty even further by suggesting the optimal number of probing points and their distribution. In this way, it should be possible to definitely design the most optimal measurement strategy.

Acknowledgments Reported research were realized within confines of projects financed by Polish National Center for Research and Development No: 0869/R/T022010/10/and LIDER/06/117/L-3/11/NCBR/2012.

Open Access This article is distributed under the terms of the Creative Commons Attribution License which permits any use, distribution, and reproduction in any medium, provided the original author(s) and the source are credited.

\section{References}

1. Barini EM, Tosello G, De Chiffre L (2010) Uncertainty analysis of pointby-point sampling complex surfaces using touch probe CMMs: DOE for complex surfaces verification with CMM. Precis Eng 34(1):16-21

2. Beaman J, Morse E (2010) Experimental evaluation of software estimates of task specific measurement uncertainty for CMMs. Precis Eng 34(1):28-33

3. Bleys P, Van Gestel N, Cuypers S, Kruth JP (2007) Uncertainty due to limited sampling of circular features on coordinate measuring machine. Proc. of the 10th CIRP Conference on Computer Aided Tolerancing, Erlangen

4. Jakubiec W (2008) Analytical determination of uncertainty of measurement in coordinate measuring technique, Scientific Dissertations 22, University of Bielsko-Biala

5. Germani M, Mandorli F, Mengoni M, Raffaeli R (2010) CAD-based environment to bridge the gap between product design and tolerance control. Precis Eng 34(1):7-15

6. Geus DA (1998) Comparison of measurement data from 3Dmeasurement in metrology for quality control in production. Proc. of 6th ISMQC IMEKO Symposium - Wien, Austria, September 8-10

7. Pedone P, Romano D (2011) Designing small samples for form error estimation with coordinate measuring machines. Precis Eng 35(2):262 270

8. Phillips SD, Borchardt B, Abackerli AJ, Shakarji C, Sawyer D (2003) The validation of CMM task specific measurement uncertainty software, Proc. of the ASPE 2003 summer topical metting "Coordinate Measuring Machines" Charlotte, North Carolina June 25-26

9. Ramu P, Yagüe JA, Hocken RJ, Miller J (2011) Development of a parametric model and virtual machine to estimate task specific measurement uncertainty for a five-axis multi-sensor coordinate measuring machine. Precis Eng 35(3):431-439

10. Schwenke H, Neuschaefer-Rube U, Pfeifer T, Kunzmann H (2002) Optical methods for dimensional metrology in production engineering. CIRP Ann Manuf Technol 51(2):685-699

11. Wilhelm RG, Hocken RJ, Schwenke H (2001) Task specific uncertainty in coordinate measurement. CIRP Ann Manuf Technol 50(2):553-563

12. Schwenke H, Knapp W, Haitjema H, Weckenmann A, Schmitt R, Delbressine F (2008) Geometric error measurement and compensation of machines - an update. CIRP Ann 57(2):660-675

13. Hocken RJ, Simpson J, Borchardt B, Lazar J, Stein P (1977) Three dimensional metrology. Ann CIRP 28:403-408

14. Sartori S, Zhang GX (1995) Geometric error measurement and compensation of machines. Ann CIRP 44(2)

15. Evans CJ (1989) Precision engineering: an evolutionary view. Cranfield Press, Cranfield
16. Hocken RJ, Pereira PH (2012) Coordinate measuring machines and systems second edition (2012) CRC Press

17. Aggogeri F, Barbato G, Modesto Barini E, Genta G, Levi R (2011) Measurement uncertainty assessment of coordinate measuring machines by simulation and planned experimentation. CIRP J Manuf Sci Technol

18. Giusca CL, Leach RK, Forbes AB (2011) A virtual machine-based uncertainty evaluation for a traceable areal surface texture measuring instrument. Measurement 44(5):988-993

19. Härtig F (1997) How to work with the on-line virtual CMM, Proc. of Workshop on Tracebility of CMM, PTB, Braunschweig, October 9-10

20. Mailhe J, Linares JM, Sprauel JM, Bourdet P (2008) Geometrical checking by virtual gauge, including measurement uncertainties. CIRP Ann Manuf Technol 57:513-516

21. Maihle J, Linares JM, Sprauel JM (2009) The statistical gauge in geometrical verification: Part I. Field of probability of the presence of matter. Precis Eng 33(4):333-341

22. Maihle J, Linares JM, Sprauel JM (2009) The statistical gauge in geometrical verification. Part II. The virtual gauge and verification process. Precis Eng 33(4):342-352

23. Oberkampf WL, DeLand SM, Rutherford BM, Diegert KV, Alvin KF (2002) Error and uncertainty in modeling and simulation. Reliab Eng Syst Saf 75(3):333-357

24. Ostrowska K (2009) Methods of evaluation of accuracy of measurements performed on coordinate measuring arms, Ph.D. Dissertation, Cracow University of Technology

25. Sładek J, Krawczyk M, Ostrowska K, Gaska A (2008) Usage of Monte Carlo method in estimation of uncertainty of coordinate measurements in example of multiple measurement method, Scientific Books ATH Bielsko Biala no 81-2008

26. Sładek J, Kupiec R, Gąska A, Kmita A (2010) Modeling of uncertainty changes caused by temperature with use of Monte Carlo method. Meas Autom Monit 01

27. Sładek J, Ostrowska K, Gąska A (2010) Virtual portable arm. Meas Autom Monit 01

28. Sładek J (2011) Accuracy of coordinate measurement. Cracow University of Technology Publishing House, Cracow

29. Sładek J (2001) Modeling and evaluation of coordinate measuring machines accuracy. Cracow University of Technology Publishing House, Cracow

30. Sładek J, Krawczyk M, Gawlik K (2007) The assessment of the coordinate measurement accuracy based on Matrix Method with use of artificial neural networks. Proc. of 10th CIRP Conference on Computer Aided Tolerancing, Erlangen

31. Summerhays KD, Baldwin JM, Campbell DA, Henke RP (2004) Application of simulation software to coordinate measurement uncertainty evaluation. Proc. of Aspe "Uncertainty Analysis in Measurement and Design"

32. Trapet E, Franke M, Hartig F, Schwenke H, Wadele F, Cox M, Forbers A, Delbressine F, Schellekens P, Trenk M, Meyer H, Morltz G, Guth T, Wanner N (1999) Traceability of coordinate measuring machines according to the method of the virtual measuring machines, PTB F-35, Braunschweig, Germany

33. Trenk M, Franke M, Schwenke H (2004) The "Virtual CMM", a software tool for uncertainty evaluation - practical application in an accredited calibration Lab. Proc. of ASPE: Uncertainty Analysis in Measurement and Design

34. Gaska A (2011) Modeling of accuracy of coordinate measurement with use of Monte Carlo method, PhD Dissertation, Cracow University of Technology, available at http://suw.biblos.pk.edu.pl/resources/i5/i2/i3/i4/ r5234/GaskaA ModelowanieDokladnosci.pdf (in Polish)

35. Huo D, Marapoulos PG, Cheng CH (2010) The framework of the virtual laser tracker - a systematic approach to the assessment of error sources and uncertainty in laser tracker measurement. Proc. of the 6th CIRP-Sponsored International Conference on Digital Enterprise Technology, part II, 507-523 
36. Sładek J, Gąska A, Olszewska M, Kupiec R, Krawczyk M (2013) Virtual coordinate measuring machine built using LaserTracer system and spherical standard. Metrol Meas Syst 20(1):77-86
37. ISO 15530-3 (2011) Geometrical product specifications (GPS) Coordinate measuring machines (CMM): technique for determining the uncertainty of measurement-Part 3: Use of calibrated workpieces or measurement standards. 